\title{
AN EXTENSION OF THE CURTISS-BIRD THEORY FOR THE RHEOLOGICAL PROPERTIES OF CONCENTRATED POLYMER SOLUTIONS AND MELTS
}

\section{BERNARD J. GEURTS}

Center for Theoretical Physics, Twente University, P.O. Box 217, Enschede 7500 AE (The Netherlands)

(Received 30 July, 1987; in revised form 31 March, 1988)

\section{Summary}

The constitutive equation for the elastic reptating rope model is considered in the limit at which the rope becomes inextensible. It is shown that in this limit a constitutive equation is obtained which differs essentially from the Curtiss-Bird theory if the correlation length is much smaller than the contour length of the rope. This constitutive equation contains an extra term related to correlations between segments. The differences between this result and the Curtiss-Bird theory are considered, and consequences are indicated.

\section{Introduction}

In a series of papers [1-6] Curtiss and Bird (and Saab) developed a kinetic theory for polymer melts in which they extended the Doi and Edwards theory [7-10] using a completely different theoretical starting point. Recently [11-13] a new model was introduced (' the elastic reptating rope model') which resulted in a constitutive equation containing the Curtiss-Bird equation as a special case [13]. In this paper this new constitutive equation is considered in the limit at which the rope becomes inextensible. It is also shown that in this limit an extension of the Curtiss-Bird theory is obtained. This new constitutive equation contains an extra term due to correlations between segments. These are included in the theory since use has been made of the two segment distribution function derived in Ref. 13.

The concept of reptation, first introduced by DeGennes [14], has proved to be very useful in modeling the complex dynamics of macromolecules in melts and concentrated solutions. Curtiss and Bird treated the reptation motion of a bead-rod chain by introducing a tensorial Stokes' law for the 
hydrodynamic interaction of the chain with the medium and anisotropic Brownian motion. In this paper we extend the tube model as introduced by Doi and Edwards. The molecule is represented by a continuous space curve ('rope') of contour length $L$. This rope is thought to be confined in a tube of the same shape and about the same cross-section. Due to the relative motion of the rope with respect to the surrounding tube a frictional force density is introduced. The molecule is considered to have a correlation length $a$ which implies that any configuration of the molecule can be approximated by $N \equiv L / a$ segments of length $a$. The tube segments are considered to move affinely along with the macroscopic flow.

In a previous paper [13] the elastic reptating rope model was analysed. A new constitutive equation was derived in which both long reptation times as well as shorter characteristic times related to internal relaxation modes of the rope, were included explicitly. This constitutive equation extended the result of Curtiss and Bird [1-6]. In the present paper we derive the constitutive equation which arises as the rope becomes inextensible. Using the two segment distribution function we analyse in detail the inextensible reptating rope model. It is shown that the new constitutive equation contains the Curtiss-Bird result as a special case. In spite of the fact that we used a completely different approach (tube model versus a full phase space kinetic description) it is of interest to notice that the constitutive equation as derived by Curtiss and Bird comes out in a natural way as a limiting case of the tube model approach, i.e. as $a=L$. We only added a hydrodynamic interaction between the rope and the tube to the original tube concept of Doi and Edwards. In general, the new equation contains an extra term which is related to correlation between the segments. This term becomes relevant as $a \ll L$.

In Section 2 we derive the constitutive equation for the case in which the rope is elastic and the relative extension small. Section 3 is devoted to the limit in which the rope becomes inextensible, and the limiting constitutive equation is derived. Finally, in Section 4 we consider the essential differences between this result and the Curtiss--Bird theory and summarize our findings.

2. The polymer contribution to the stress tensor for the elastic reptating rope

In this section we derive the polymer contribution to the stress tensor $T(t)$ for the elastic reptating rope model. As was shown in Ref. 13, the polymer contribution, in the continuous limit, to the stress tensor $T(t)$ can be written as

$$
\boldsymbol{T}(t)=n N \int_{0}^{L} \mathrm{~d} s\langle\sigma(s, t) e(s, t) e(s, t)\rangle *,
$$


where $n$ is the number density of polymers, $\sigma(s, t)$ is the tension along the rope at $s$ at time $t$, and $e(s, t)$ is the unit vector tangent to the rope at $s$ at time $t$. The average denoted by $\langle\cdots\rangle^{*}$ is an ensemble average of the random variable $\sigma(s, t) e(s, t) e(s, t)$. As we will show momentarily, $\sigma(s, t)$ depends on the tangent vectors $e\left(s^{\prime}, t^{\prime}\right)$ at positions $s^{\prime}$ along the rope at previous times $t^{\prime}<t$. Hence the averaging in (2.1) will have to deal with both $e\left(s^{\prime}, t^{\prime}\right)$ as well as $e(s, t)$. So we need to simultaneous probability density for the tangent vectors at $\left(s^{\prime}, t^{\prime}\right)$ and $(s, t)$ in order to perform the averaging. This simultaneous probability density should be consistent with the independent alignment approximation adopted by Doi and Edwards. We will refer to it as the two-segment distribution function in the present paper.

The tension $\sigma(s, t)$ along the rope is determined by the force density $f$ through the equation of motion. If we disregard inertial effects and consider only small extensions the force balance on an elementary rope segment reads

$\partial_{s} \sigma(s, t)+f(s . t)=0$.

It is strictly one dimensional $(s)$, consistent with the tube model. The force density $f$ is considered to consist of two parts: a Brownian force density $f_{\mathrm{B}}$ and a frictional force density $f_{\mathrm{H}}$, related to the relative motion of the rope with respect to the tube. In the case of small extensions in the rope, these two force densities can be thought independent and the reptative diffusion of segments along the rope is not influenced by this extensibility to first order. Consistent with the reptation motion which implies random back and forth movement of the rope along its own contour, the random Brownian forces act only at the ends of the rope. The constraints on the motion of the rope imposed by the surrounding tube imply shifts of inner segments to neighboring positions induced by the creation and annihilation of the end-segments. Consequently, the Brownian force density $f_{\mathrm{B}}$ is zero along the rope and nonzero only at the end points. The resulting Brownian tension in the rope $\sigma_{\mathrm{B}}$, was shown to be given by $\sigma_{\mathrm{B}}=k T / L$ in Ref. 13 ( $k$ is Boltzmann's constant, $T$ the temperature). Due to the constraint imposed by the tube on the motion of the rope the frictional force density $f_{\mathrm{H}}$ yields a force directed along $e(s, t)$. It is taken proportional to the difference in velocity of the rope and the velocity of the tube in the direction of $e(s, t)$. As was shown in Ref. 13 the total tension $\sigma$ can then be written as

$\sigma(s, t)=k T / L+\kappa \epsilon(s, t)$.

Here $\kappa$ is the elasticity modulus of the rope. The relative extension $\epsilon(s, t)$ is defined with respect to equilibrium, i.e. if only Brownian forces act on the rope. If $X(s, t)$ denotes the position in three-dimensional space of a point 
on the rope at arc length $s$ measured with respect to one of the end points at time $t$ then the relative extension $\epsilon$ is given by

$\epsilon(s, t)=\left|\nabla_{s} X(s, t)\right|-1$,

where $\nabla_{s} X \equiv\left[\partial_{s} X_{1}, \partial_{s} X_{2}, \partial_{s} X_{3}\right]$ and $|X|$ denotes the Euclidean length of the vector $X$. Notice that at equilibrium $\epsilon=0$. Hence, the 'elastic tension' $\kappa \in(s, t)$ corresponds to an additional tension in the rope due to the hydrodynamic interaction with the medium if this medium is in a state of macroscopic deformation. This extension is determined by the equation of motion of the rope, which reduces to the diffusion equation if the relative extension is small [13]

$\partial_{t} \epsilon-\kappa \partial_{s s} \epsilon t \zeta=e(s, t) \cdot D(t) \cdot e(s, t)$,

where $\zeta$ is the friction coefficient per unit length of the rope and $D$ the macroscopic rate of strain tensor. Note that (2.5) is valid for the entire rope. We imply equilibrium conditions for $t \leq 0$, i.e. the initial situation $(t=0)$ is taken to be the unstretched rope. Since the 'elastic tension' at the ends of the rope must vanish we have as boundary conditions $\epsilon(0, t)=\epsilon(L, t)=0$. Under the assumption that during the deformations the relative variations in the contour length $L$ of the rope are small, one may derive

$\epsilon(s, t)=\int_{0}^{t} \mathrm{~d} t^{\prime} \int_{0}^{L} \mathrm{~d} s^{\prime} \nu\left(s, s^{\prime}, t-t^{\prime}\right) e\left(s^{\prime}, t^{\prime}\right) \cdot D\left(t^{\prime}\right) \cdot e\left(s^{\prime}, t^{\prime}\right)$,

where

$$
\nu\left(s, s^{\prime}, t-t^{\prime}\right)=\frac{2}{L} \sum_{m=1}^{\infty} \sin \left(\frac{m \pi s^{\prime}}{L}\right) \sin \left(\frac{m \pi s}{L}\right) \exp \left[-\frac{\left(t-t^{\prime}\right) m^{2}}{\tau_{\epsilon}}\right],
$$

with time constant $\tau_{\mathrm{e}} \equiv L^{2} \zeta / \pi^{2} \kappa\left(\tau_{\mathrm{e}}\right.$ scales as $N^{2}$ if $\kappa$ is interpreted as the modulus of a Gaussian chain. Hence for long polymers $\tau_{e}$ is much smaller than characteristic times related to the reptation process). Notice that $\epsilon$ is expressed in terms of the tangent vector at $s^{\prime}$ at time $t^{\prime}$ and the rate of strain tensor $D$ at time $t^{\prime}$. Through (2.3) and (2.6) the total tension $\sigma(s, t)$ depends on $e\left(s^{\prime}, t^{\prime}\right)$ and $D\left(t^{\prime}\right)$ as well and hence, in order to perform the averaging needed in (2.1) to obtain the stress tensor $T(t)$ one needs the probability density that simultaneously $e(s, t)$ is in some direction $e$ and $e\left(s^{\prime}, t^{\prime}\right)$ in some (other) direction $e^{\prime}$. This two segment average will be discussed shortly. Combining (2.6) and (2.3) and inserting this into (2.1) yields for $T(t)$ after some calculation

$$
\begin{aligned}
T(t)= & n N k T\left[\int_{0}^{L} \mathrm{~d} s \frac{1}{L}\langle e(s, t) e(s, t)\rangle_{1}\right. \\
& +\frac{1}{k T} \int_{0}^{t} \mathrm{~d} t^{\prime} D\left(t^{\prime}\right): \int_{0}^{L} \mathrm{~d} s \int_{0}^{L} \mathrm{~d} s^{\prime} \kappa \nu\left(s, s^{\prime}, t-t^{\prime}\right) \\
& \left.\times\left\langle e\left(s^{\prime}, t^{\prime}\right) e\left(s^{\prime}, t^{\prime}\right) e(s, t) e(s, t)\right\rangle_{2}\right]
\end{aligned}
$$


The average $\langle e(s, t) e(s, t)\rangle_{1}$ involves the one segment distribution function $\Psi(e ; s, t)$ as derived by Doi and Edwards [9]. Within the independent alignment approximation it is given by

$\Psi(e ; s, t)=\int_{-\infty}^{t} \mathrm{~d} t^{\prime} \chi\left(s, t-t^{\prime}\right) \int \mathrm{d} e^{\prime} \frac{1}{4 \pi} \delta\left(e-g\left(t, t^{\prime}, e^{\prime}\right)\right)$,

where

$\chi\left(s, t-t^{\prime}\right)=\sum_{m=0}^{\infty} \frac{4(2 m+1)}{\pi \tau_{\mathrm{d}}} \sin \left(\frac{(2 m+1) \pi s}{L}\right) \exp \left[-\frac{\left(t-t^{\prime}\right)(2 m+1)^{2}}{\tau_{\mathrm{d}}}\right]$,

in which $\tau_{\mathrm{d}} \equiv L^{2} / \pi^{2} D$ is the 'disengagement time' as introduced by Doi and Edwards. The diffusion coefficient $D$ which appears here characterizes the reptative diffusion motion of segments along the rope (it scales as $N^{-1}$ if $N \gg 1$ [14]). The operator $g$ is given by $g\left(t, t^{\prime}, e^{\prime}\right) \equiv F_{t^{\prime}}(t) \cdot e^{\prime} /\left|F_{t}(t) \cdot e^{\prime}\right|$ where $F_{t^{\prime}}(t)$ is the macroscopic deformation gradient. Evaluation of this average yields exactly the Doi and Edwards result for the first term in (2.8).

The evaluation of the average $\left\langle e\left(s^{\prime}, t^{\prime}\right) e\left(s^{\prime}, t^{\prime}\right) e(s, t) e(s, t)\right\rangle_{2}$ is quite complicated. The two segment distribution function $\Phi\left(e, e^{\prime} ; s, t, s^{\prime}, t^{\prime}\right)$ which is consistent with the independent alignment assumption is needed. It is defined by

$\Phi\left(e, e^{\prime} ; s, t, s^{\prime}, t^{\prime}\right)=\operatorname{Pr}\left(e(s, t)=e, e\left(s^{\prime}, t^{\prime}\right)=e^{\prime}\right)$,

that is the probability that $e(s, t)=e$ and $e\left(s^{\prime}, t^{\prime}\right)=e^{\prime}$. By definition one has

$$
\begin{aligned}
& \operatorname{Pr}\left(\boldsymbol{e}(s, t)=e, e\left(s^{\prime}, t^{\prime}\right)=e^{\prime}\right)=\operatorname{Pr}\left(e\left(s^{\prime}, t^{\prime}\right)=e^{\prime}\right) \\
& \times \operatorname{Pr}\left(e(s, t)=e \mid e\left(s^{\prime}, t^{\prime}\right)=e^{\prime}\right)
\end{aligned}
$$

and clearly, within the independent alignment approximation,

$\operatorname{Pr}\left(e\left(s^{\prime}, t^{\prime}\right)=e^{\prime}\right)=\Psi\left(e^{\prime} ; s^{\prime}, t^{\prime}\right) \mathrm{d} e^{\prime}$.

So we only need to specify the conditional probability that $e(s, t)=e$ if $e\left(s^{\prime}, t^{\prime}\right)=e^{\prime}$. This latter probability has two contributions. First, consistent with the tube model, the reptation concept pictures that within the course of time endsegments are created and annihilated continuously. So the probability that $e(s, t)=e$ is partly determined by these endsegments. The endsegments created between $t^{\prime \prime}$ and $t^{\prime \prime}+\mathrm{d} t^{\prime \prime}$ evolve under the reptation process and may occupy the position labeled $s$ at time $t$ with probability $\chi(\mathrm{s}, t-$ $\left.t^{\prime \prime}\right) \mathrm{d} t^{\prime \prime}$. The original direction $e^{\prime \prime}$ of the endsegment created at time $t^{\prime \prime}$ has transformed affinely along with the macroscopic flow and may be directed in the desired direction $e$. Second, there is a similar contribution coming 
from all the interior segments $e\left(s^{\prime \prime}, t^{\prime}\right)$ at time $t^{\prime}$. These can also be taken to $s$ at time $t$ through the reptative diffusion along the rope and may have acquired the desired direction $e$. The condition that $e\left(s^{\prime}, t^{\prime}\right)=e^{\prime}$ only influences this contribution. Clearly the distribution of $e\left(s^{\prime \prime}, t^{\prime}\right)$ is influenced by the condition that at $s^{\prime}$ at time $t^{\prime}$ the segment is in the presumed fixed position $e^{\prime}$. As $\left|s^{\prime \prime}-s^{\prime}\right|>a$ the segments will be taken independent and as $\left|s^{\prime \prime}-s^{\prime}\right|<a$ one has the direction $e^{\prime}$ by the conditional probability in (2.12). As $t-t^{\prime} \gg \tau_{\mathrm{d}}$ then the main contribution comes from the end-segments and the conditional probability in (2.12) approaches asymptotically $\Psi(e ; s, t)$ de. However, as $t-t^{\prime}$ is of the order of $\tau_{\mathrm{d}}$ exactly the second contribution becomes dominant. The details of these contributions can be found in Ref. 13. After a lengthy calculation (see Ref. 13) one obtains for the average

$$
\begin{aligned}
& \left\langle\boldsymbol{e}\left(s^{\prime}, t^{\prime}\right) e\left(s^{\prime}, t^{\prime}\right) e(s, t) e(s, t)\right\rangle_{2} \\
& =\left\langle e\left(s^{\prime}, t^{\prime}\right) e\left(s^{\prime}, t^{\prime}\right)\right\rangle_{1}\langle e(s, t) e(s, t)\rangle_{1} \\
& \quad-\left\langle e\left(s^{\prime}, t^{\prime}\right) e\left(s^{\prime}, t^{\prime}\right)\right\rangle_{1}\left\langle e\left(s, t^{\prime}\right) e\left(s, t^{\prime}\right)\right\rangle_{1} \\
& \quad+\left\langle e\left(s^{\prime}, t^{\prime}\right) e\left(s^{\prime}, t^{\prime}\right) g\left(t, t^{\prime}, e\left(s^{\prime}, t^{\prime}\right)\right) g\left(t, t^{\prime}, e\left(s^{\prime}, t^{\prime}\right)\right)\right\rangle_{1} \\
& \quad \times \int_{\max \left(0, s^{\prime}-a\right)}^{\min \left(L, s^{\prime}+a\right)} \mathrm{d} s^{\prime \prime} q\left(t-t^{\prime}, s^{\prime \prime}, s\right) \\
& \quad+\left\langle e\left(s^{\prime}, t^{\prime}\right) e\left(s^{\prime}, t^{\prime}\right)\right\rangle_{1} f_{0}^{L} \mathrm{~d} s^{\prime \prime} q\left(t-t^{\prime}, s^{\prime \prime}, s\right) \\
& \quad \times\left\langle g\left(t, t^{\prime}, e\left(s^{\prime \prime}, t^{\prime}\right)\right) g\left(t, t^{\prime}, e\left(s^{\prime \prime}, t^{\prime}\right)\right)\right\rangle_{1},
\end{aligned}
$$

where

$$
f_{0}^{L} \mathrm{~d} s^{\prime \prime} \equiv \int_{0}^{\max \left(0, s^{\prime}+a\right)} \mathrm{d} s^{\prime \prime}+\int_{\min \left(L, s^{\prime}+a\right)}^{L} \mathrm{~d} s^{\prime \prime} .
$$

The function $q$ which appears here is the transition probability density for a segment which was at $s^{\prime \prime}$ at time $t^{\prime}$ to be at $s$ at time $t$. It is given in Ref. 13 and is related to a first passage problem with two absorbing boundaries [15]. The averages on the right hand side in (2:14) are with respect to the one segment distribution function $\Psi$, taken at the appropriate position and time variables.

The set of equations (2.8-2.15) together constitute an implicit prescription from which the stress tensor $\boldsymbol{T}$ can be obtained. In it we have included explicitly short characteristic times (cf. $\tau_{\epsilon}$ through $\epsilon$ ) related to internal relaxation modes in the rope, as well as long reptation times (cf. $\tau_{\mathrm{d}}$ included through the one segment probability density $\Psi$ ).

In the next section we will consider the limit in which the rope becomes inextensible, i.e. we consider the limit $\kappa \rightarrow \infty$. As will be clear from (2.8) this 
limit only affects the second term in (2.8), which represents the contribution due to the hydrodynamic interaction. As $\kappa$ increases, the rheological behavior becomes more and more dominated by the deformations which occurred just before the present time since $\tau_{\epsilon} \ll 1$ in this case so that the function $\nu$ in (2.8) becomes sharply peaked around $t^{\prime}=t$ and hence $\epsilon(s, t)$ is mainly determined by $e \cdot D \cdot e$ for $t^{\prime} \approx t$ (cf. (2.6)). This simplifies the $\kappa \rightarrow \infty$ limit theory greatly and an explicit constitutive equation can be derived.

\section{The constitutive equation for the inextensible reptating rope}

In this section we consider the limit $\kappa \rightarrow \infty$, in which case the rope becomes inextensible. First we derive the polymer contribution to the stress tensor for general correlation length $a$. Then we consider in more detail the cases $a=L$ and $a=0$. As will be clear, we only need to focus on the two segment average in this limit. The one segment average yields the Doi and Edwards constitutive equation.

For convenience we introduce the following notation

$\boldsymbol{H}\left(s^{\prime}, t^{\prime}, s, t\right) \equiv \boldsymbol{D}\left(t^{\prime}\right):\left\langle e\left(s^{\prime}, t^{\prime}\right) e\left(s^{\prime}, t^{\prime}\right) e(s, t) e(s, t)\right\rangle_{2}$.

If we transform the integration variable $t^{\prime}$ in (2.8) as

$z=\frac{m^{2}}{\tau_{\epsilon}}\left(t-t^{\prime}\right)$

and set $x=s / L$ and $x^{\prime}=s^{\prime} / L$, it is easy to show that the second term between brackets in (2.8) can be written as

$$
\begin{aligned}
& \tau_{\mathrm{d}} \int_{0}^{1} \mathrm{~d} x \int_{0}^{1} \mathrm{~d} x^{\prime} 2 \sum_{m=1}^{\infty} \frac{1}{m^{2}} \sin \left(m \pi x^{\prime}\right) \\
& \quad \times \sin (m \pi x) \int_{0}^{m^{2} t / \tau_{c}} \mathrm{~d} z e^{-z} \boldsymbol{H}\left(L x^{\prime}, t-\frac{\tau_{\varepsilon} z}{m^{2}}, L x, t\right) .
\end{aligned}
$$

Since $\tau_{\epsilon} \rightarrow 0$ as $\kappa \rightarrow \infty$ one has for all $t>0$

$$
\lim _{\kappa \rightarrow 0} \int_{0}^{m^{2} t / \tau_{\varepsilon}} \mathrm{d} z e^{-z} \boldsymbol{H}\left(L x^{\prime}, t-\frac{\tau_{\epsilon} z}{m^{2}}, L x, t\right)=\boldsymbol{H}\left(L x^{\prime}, t, L x^{\prime}, t\right) .
$$

Hence if we take the limit $\kappa \rightarrow \infty$ under the integration, we may write (3.3) as

$$
\tau_{\mathrm{d}} \int_{0}^{1} \mathrm{~d} x \int_{0}^{1} \mathrm{~d} x^{\prime} f\left(x, x^{\prime}\right) H\left(L x^{\prime}, t, L x, t\right),
$$

where

$$
f\left(x, x^{\prime}\right)=2 \sum_{m=1}^{\infty} \frac{1}{m^{2}} \sin (m \pi x) \sin \left(m \pi x^{\prime}\right) .
$$


It is not hard to prove that

$$
\begin{aligned}
f\left(x, x^{\prime}\right) & =\pi^{2} x\left(1-x^{\prime}\right) & ; 0 \leq x \leq x^{\prime}, \\
& =\pi^{2} x^{\prime}(1-x) & ; x^{\prime} \leq x \leq 1,
\end{aligned}
$$

and in terms of $s$ and $s^{\prime}$ one has by inspection of (2.14)

$$
\begin{aligned}
H\left(s^{\prime}, t, s, t\right)= & \boldsymbol{D}(t):\left[\left\langle e\left(s^{\prime}, t\right) e\left(s^{\prime}, t\right) e\left(s^{\prime}, t\right) e\left(s^{\prime}, t\right)\right\rangle_{1}\right. \\
& \times \int_{\max \left(0, s^{\prime}-a\right)}^{\min \left(L, s^{\prime}+a\right)} \mathrm{d} s^{\prime \prime} \delta\left(s-s^{\prime \prime}\right) \\
& \left.+\left\langle e\left(s^{\prime}, t\right) e\left(s^{\prime}, t\right)\right\rangle_{1} f_{0}^{L} \mathrm{~d} s^{\prime \prime}\left\langle e\left(s^{\prime \prime}, t\right) e\left(s^{\prime \prime}, t\right)\right\rangle_{1} \delta\left(s-s^{\prime \prime}\right)\right]
\end{aligned}
$$

The $\delta$-function appears here because [15]

$$
\lim _{t^{\prime} \rightarrow t} q\left(t-t^{\prime}, s^{\prime \prime}, s\right)=\delta\left(s^{\prime \prime}-s\right) \text {. }
$$

Upon evaluating the integrals in (3.9) one finds

$$
\begin{aligned}
\boldsymbol{H}\left(L x^{\prime}, t, L x, t\right)= & D(t):\left[\left\langle e\left(L x^{\prime}, t\right) e\left(L x^{\prime}, t\right) e\left(L x^{\prime}, t\right) e\left(L x^{\prime}, t\right)\right\rangle_{1}\right. \\
& \times\left(\vartheta\left(x-\max \left(0, x^{\prime}-\Delta\right)\right)-\vartheta\left(x-\min \left(1, x^{\prime}+\Delta\right)\right)\right) \\
& +\left\langle e\left(L x^{\prime}, t\right) e\left(L x^{\prime}, t\right)\right\rangle_{1} \\
& \times\langle e(L x, t) e(L x, t)\rangle_{1}\left(1-\left(\vartheta\left(x-\max \left(0, x^{\prime}-\Delta\right)\right)\right.\right. \\
& \left.\left.\left.-\vartheta\left(x-\min \left(1, x^{\prime}+\Delta\right)\right)\right)\right)\right]
\end{aligned}
$$

where

$$
\begin{aligned}
\vartheta(x)=1 & ; x \geq 0, \\
=0 & ; x<0 .
\end{aligned}
$$

and the reduced correlation length parameter $\Delta \equiv a / L$. Combination of (3.4-13) yields after some calculation for (3.3)

$$
\begin{aligned}
& D(t):\left[\int_{-\infty}^{t} \mathrm{~d} t^{\prime} A\left(t-t^{\prime} ; \Delta\right)\langle\text { eeee }\rangle_{0}\left(t, t^{\prime}\right)\right. \\
& \left.\quad+\int_{-\infty}^{t} \mathrm{~d} t^{\prime} \int_{-\infty}^{t} \mathrm{~d} t^{\prime \prime} B\left(t-t^{\prime}, t-t^{\prime \prime} ; \Delta\right)\langle e e\rangle_{0}\left(t, t^{\prime}\right)\langle e e\rangle_{0}\left(t, t^{\prime \prime}\right)\right],
\end{aligned}
$$

where

$$
\begin{aligned}
& \langle e e\rangle_{0}(\alpha, \beta)=\int \mathrm{d} e^{\prime} \frac{1}{4 \pi} g\left(\alpha, \beta, e^{\prime}\right) g\left(\alpha, \beta, e^{\prime}\right) \\
& \langle\text { eеee }\rangle_{0}(\alpha, \beta)=\int \mathrm{d} e^{\prime} \frac{1}{4 \pi} g\left(\alpha, \beta, e^{\prime}\right) g\left(\alpha, \beta, e^{\prime}\right) g\left(\alpha, \beta, e^{\prime}\right) g\left(\alpha, \beta, e^{\prime}\right)
\end{aligned}
$$


are the averages over the unit sphere with respect to the uniform distribution function. This uniform distribution function expresses the fact that endsegments are created in arbitrary directions with equal probability [14]. The weightfunctions $A$ and $B$ are given by

$$
\begin{aligned}
& A\left(t-t^{\prime} ; \Delta\right)=\sum_{m=0}^{\infty} \frac{8}{\pi^{2}(2 m+1)^{2}} \exp \left(-\frac{\left(t-t^{\prime}\right)(2 m+1)^{2}}{\tau_{\mathrm{d}}}\right) \alpha_{m}(\Delta) \\
& B\left(t-t^{\prime}, t-t^{\prime \prime} ; \Delta\right)=\sum_{m=0}^{\infty} \sum_{p=0}^{\infty} \frac{16(2 m+1)(2 p+1)}{\tau_{\mathrm{d}}} \\
& \times \exp \left(-\frac{\left(t-t^{\prime}\right)(2 m+1)^{2}}{\tau_{\mathrm{d}}}\right) \\
& \times \exp \left(-\frac{\left(t-t^{\prime \prime}\right)(2 p+1)^{2}}{\tau_{\mathrm{d}}}\right) \beta_{m, p}(\Delta) .
\end{aligned}
$$

The combinatorial factors $\alpha_{m}(\Delta)$ and $\beta_{m, p}(\Delta)$ can be shown to be given by $\alpha_{m}(\Delta)=\frac{\pi^{3}(2 m+1)^{3}}{2} \int_{0}^{1} \mathrm{~d} x^{\prime} J_{\alpha}\left(x^{\prime} ; \Delta\right) \sin \left((2 m+1) \pi x^{\prime}\right)$,

where $J_{\alpha}\left(x^{\prime} ; \Delta\right)$ is given by

$$
\begin{aligned}
J_{\alpha}\left(x^{\prime} ; \Delta\right)= & -\frac{1}{2} x^{\prime 2}-\frac{1}{2}\left[\max \left(0, x^{\prime}-\Delta\right)\right]^{2}\left(1-x^{\prime}\right) \\
& +x^{\prime} \min \left(1, x^{\prime}+\Delta\right)\left[1-\frac{1}{2} \min \left(1, x^{\prime}+\Delta\right)\right] .
\end{aligned}
$$

Also

$\beta_{m, p}(\Delta)=\int_{0}^{1} \mathrm{~d} x^{\prime} J_{\beta, m}\left(x^{\prime} ; \Delta\right) \sin \left((2 p+1) \pi x^{\prime}\right)$,

where

$$
\begin{aligned}
J_{\beta, m}\left(x^{\prime} ; \Delta\right)= & \left(1-x^{\prime}\right) \int_{0}^{\max \left(0, x^{\prime}-\Delta\right)} \mathrm{d} x x \sin ((2 m+1) \pi x) \\
& +x^{\prime} \int_{\min \left(1, x^{\prime}+\Delta\right)}^{1} \mathrm{~d} x(1-x) \sin ((2 m+1) \pi x) .
\end{aligned}
$$

After a lengthy calculation, performed partly with the manipulation program REDUCE [16] one obtains explicitly

$$
\begin{gathered}
\alpha_{m}(\Delta)=[2 \Delta-1]+[1-\Delta] \cos ((2 m+1) \pi \Delta) \\
+\frac{3}{\pi(2 m+1)} \sin ((2 m+1) \pi \Delta),
\end{gathered}
$$

$\beta_{m, m}(\Delta)=(1-\Delta) \frac{\sin (m \pi \Delta)}{2 m^{3} \pi^{3}}+(1-\Delta)^{2} \frac{\cos (m \pi \Delta)}{2 m^{2} \pi^{2}}-(1-\Delta)^{3} \frac{\sin (m \pi \Delta)}{6 m \pi}$ 
and

$$
\begin{aligned}
\beta_{m, p}(\Delta)= & \frac{16 m p}{\pi^{4}\left(m^{2}-p^{2}\right)^{3}}[\cos (p \pi \Delta)-\cos (m \pi \Delta)] \\
& +(1-\Delta) \frac{4(m \sin (p \pi \Delta)+p \sin (m \pi \Delta))}{\pi^{3}\left(m^{2}-p^{2}\right)^{2}}
\end{aligned}
$$

where it is understood that $m$ and $p$ on the right hand sides of $(3.24,25)$ can take on odd positive values only. Notice that $\beta_{m, p}(\Delta)=\beta_{p, m}(\Delta)$.

The total polymer contribution to the stress tensor can now be written as

$$
\begin{aligned}
T(t)= & n N k T\left[\int_{-\infty}^{t} \mathrm{~d} t^{\prime} \mu\left(t-t^{\prime}\right)\langle e e\rangle_{0}\left(t, t^{\prime}\right)\right. \\
& +D(t): \int_{-\infty}^{t} \mathrm{~d} t^{\prime} A\left(t-t^{\prime} ; \Delta\right)\langle\text { eeee }\rangle_{0}\left(t, t^{\prime}\right) \\
& +D(t): \int_{-\infty}^{t} \mathrm{~d} t^{\prime} \int_{-\infty}^{t} \mathrm{~d} t^{\prime \prime} B\left(t-t^{\prime}, t-t^{\prime \prime} ; \Delta\right) \\
& \left.\times\langle e e\rangle_{0}\left(t, t^{\prime}\right)\langle e e\rangle_{0}\left(t, t^{\prime \prime}\right)\right],
\end{aligned}
$$

where

$\mu\left(t-t^{\prime}\right)=\frac{1}{L} \int_{0}^{L} \mathrm{~d} s \chi\left(s, t-t^{\prime}\right)=\sum_{m=0}^{\infty} \frac{8}{\pi^{2} \tau_{\mathrm{d}}} \exp \left(-\frac{\left(t-t^{\prime}\right)(2 m+1)^{2}}{\tau_{\mathrm{d}}}\right)$.

It contains three different terms. The first term is exactly equal to the constitutive equation of Doi and Edwards. The second term has the same form as the extra term first derived by Curtiss and Bird. However the weightfunction $A$ is slightly different from their corresponding weightfunction. We discuss these differences shortly. The third term in (3.26) is essentially new. In appearance it resembles a 'quadratic' form of the Doi and Edwards term. It has entered the theory since we used the two segment distribution function. As we remarked before, $\kappa \rightarrow \infty$ implies that the rheological behavior is mainly determined by the deformations which occurred just before the present time. However, in that case the contribution of the endsegments to the conditional probability in (2.12) becomes very small, simply because the reptative diffusion process has not moved the endsegments far enough to contribute to the probability that $e(s, t)=e$, for most $s$-values. Hence, since we implied the condition that $e\left(s^{\prime}, t^{\prime}\right)=e^{\prime}$ in (2.12) the probability density for the random variable $e\left(s^{\prime}, t\right)$ will be sharply peaked around $e^{\prime}$ for the terms that contribute mainly to the stress tensor 
(since $t-t^{\prime} \leq \tau_{d}$ ). This of course is completely different from the one segment distribution function and results after careful calculation in the new third term in this constitutive equation. We will now consider some properties of the weighting functions $A$ and $B$ for future reference.

As one may easily verify, as $\Delta=1$, the weighting function $A$ reduces to the one given by Curtiss and Bird, i.e. since all $\alpha_{m}(1)=1$

$A\left(t-t^{\prime} ; 1\right)=\sum_{m=0}^{\infty} \frac{8}{\pi^{2}(2 m+1)^{2}} \exp \left[-\frac{\left(t-t^{\prime}\right)(2 m+1)^{2}}{\tau_{\mathrm{d}}}\right]$.

In addition the long time behavior $\left(t-t^{\prime} \gg \tau_{d}\right)$ is easily seen to be independent of $\Delta$. Hence, contributions of deformation processes which occurred long ago are all weighed in the same manner as in the Curtiss-Bird theory. For short times $\left(t-t^{\prime} \ll \tau_{\mathrm{d}}\right)$, the function $A$ varies significantly with $\Delta$. To illustrate this, let us consider $A(0 ; \Delta)$ which is given by

$$
\begin{aligned}
A(0 ; \Delta)= & \frac{8}{\pi^{2}}\left[(2 \Delta-1) \sum_{m=0}^{\infty} \frac{1}{(2 m+1)^{2}}+(1-\Delta) \sum_{m=0}^{\infty} \frac{\cos ((2 m+1) \pi \Delta)}{(2 m+1)^{2}}\right. \\
& \left.+\frac{3}{\pi} \sum_{m=0}^{\infty} \frac{\sin ((2 m+1) \pi \Delta)}{(2 m+1)^{3}}\right] .
\end{aligned}
$$

It is not hard to prove that

$$
\begin{aligned}
& \sum_{m=0}^{\infty} \frac{\cos ((2 m+1) \pi \Delta)}{(2 m+1)^{2}}=\frac{\pi^{2}}{8}(1-2 \Delta), \\
& \sum_{m=0}^{\infty} \frac{\sin ((2 m+1) \pi \Delta)}{(2 m+1)^{3}}=\frac{\pi^{3}}{8} \Delta(1-\Delta),
\end{aligned}
$$

so, since summing $(2 m+1)^{-2}$ yields $\pi^{2} / 8$ one finds

$A(0 ; \Delta)=\Delta(2-\Delta)$.

Taking $A(0 ; 1)-A(0 ; \Delta)$ as a measure for the deviation from the Curtiss and Bird theory, we see that as $\Delta$ is decreased from 1 , it has to be quite small before this quantity starts to build up significantly. A feature that does not become clear from this analysis is the fact that for $\Delta<1 / 2$ the function $A$ has a maximum for $t-t^{\prime}>0$ rather than for $t=t^{\prime}$, which is the case as $\Delta>1 / 2$. This is shown in Fig. 1 , and it implies that as $\Delta$ is small enough, processes which occurred somewhat before the present time have a slightly higher weight than processes which occurred directly before the present time. This is due to the fact that $\alpha_{m}(\Delta)$ is a fast oscillating function for large $m$, which can be negative for small $\Delta$-values. As $\Delta$ is increased the 


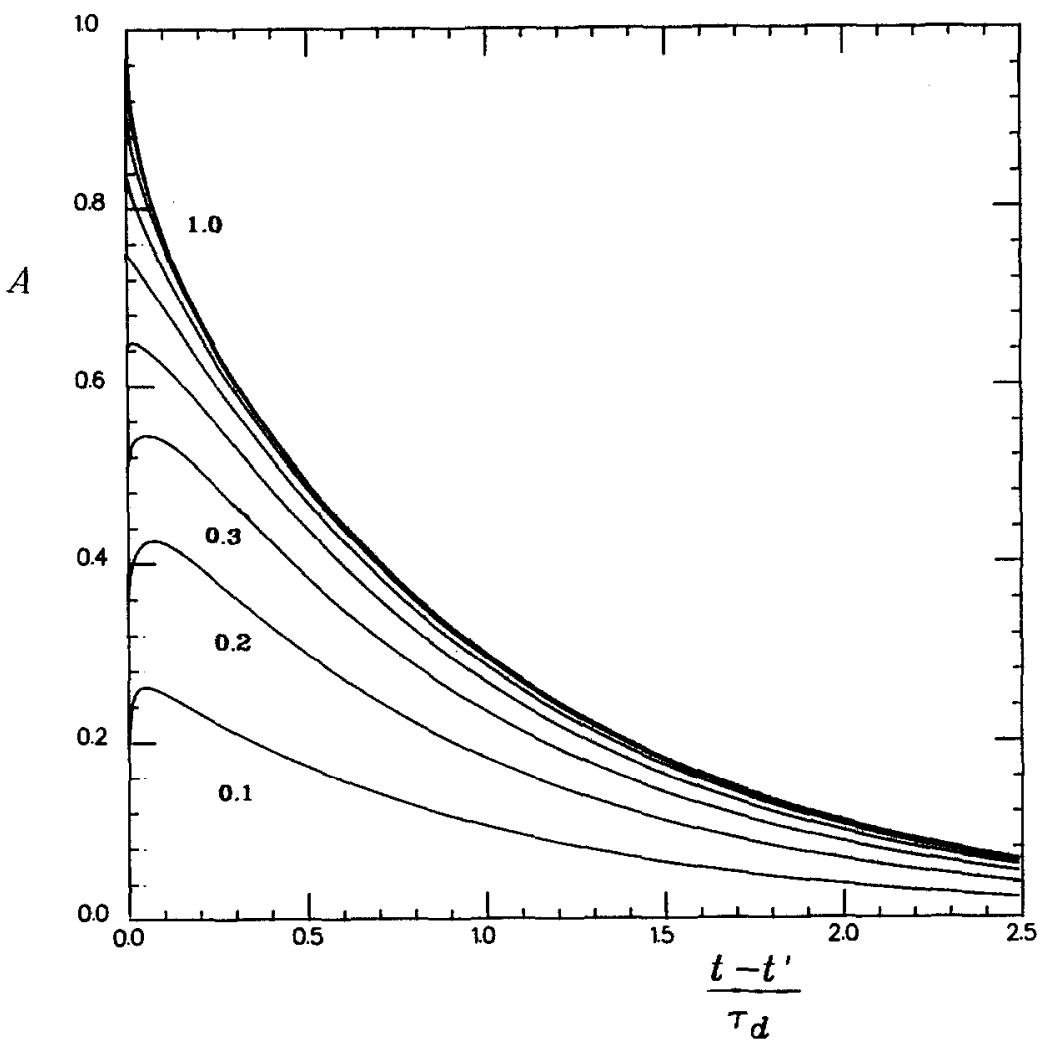

Fig. 1. The weighting function $A\left(t-t^{\prime} ; \Delta\right)$ for various values of the parameter $\Delta$. The value of $\Delta$ is indicated on the corresponding line.

amplitude of the oscillations decreases and for sufficiently high $\Delta, \alpha_{m}(\Delta)$ is strictly positive for all $\mathrm{m}$.

The weighting function $B$ is much more difficult to study. One may easily verify that as $\Delta=1, B=0$ and since $A$ reduces to the Curtiss-Bird result in this case, we notice that our constitutive equation reduces exactly to theirs, with 'link tension coefficient' equal to $1 / 2$, in this limit. The value $1 / 2$ for the link tension coefficient has already been derived for the reptating rope model in Refs. 11 and 12. With this value for the link tension coefficient the Curtiss-Bird theory showed to give good agreement with a large variety of experimental data [3-5]. As $\Delta=0$ one has

$\beta_{m, p}=\frac{1}{2(2 m+1)^{2} \pi^{2}} \delta_{m, p}$,

where $\delta_{m, p}$ is the Kronecker delta. This implies that

$B\left(t-t^{\prime}, t-t^{\prime \prime} ; 0\right)=\mu\left(\left[t-t^{\prime}\right]+\left[t-t^{\prime \prime}\right]\right)$, 
where $\mu$ is given by (3.27). The divergence of $B$ as $t \rightarrow t^{\prime}, t \rightarrow t^{\prime \prime}$, is thus expected to be of the same type as for $\mu$ (see Refs. 1-6). Finally, since the new third term in (3.26) is of a quadratic nature one may expect that the contribution of this term to the stress tensor will be small as $\Delta$ is quite large $(>1 / 2)$. Hence, the deviations from the Curtiss-Bird theory will only be significant for relatively low $\Delta$.

\section{Concluding remarks}

We considered the elastic reptating rope model [13] in the limit in which the rope becomes inextensible. A new constitutive equation was derived (cf. eqn. (3.26)) which extends the equation obtained by Curtiss and Bird [1-6]. As was shown, their result, with link tension coefficient equal to $1 / 2$, is exactly reproduced if the correlation length $a$ is equal to the contour length $L$ of the rope. It is remarkable that a completely different theoretical approach yields in this limit the same constitutive equation. Our approach is very similar to that of Doi and Edwards. We extended their treatment by introducing a hydrodynamic interaction between the rope and the tube, and obtained a constitutive equation that contains the Curtiss-Bird equation as a special case. As was argued in Section 3, if $a$ is of the same order as $L$, the deviations from the Curtiss-Bird theory will be small. The predictions of the rheological behavior of polymer melts have been found to be consistent with their theory as $a / L \geq 1 / 2$ and differ significantly as $a / L<1 / 2$ [17].

The new term in the constitutive equation appeared as a result of an extension of the averaging procedure in obtaining the stress tensor. Rather than using the one segment distribution function as derived by Doi and Edwards we derived a two segment distribution function consistent with the independent alignment approximation. Without this extension one would only reobtain the Curtiss and Bird constitutive equation [11].

\section{Acknowledgements}

The author would like to express his gratitude to Dr. Ruud van Damme of the Department of Computer Science of Twente University for several useful discussions and stimulating remarks. Drs. Robert J.J. Jongschaap of the Rheology Group of Twente University has stimulated me to start these calculations.

\section{References}

1 C.F. Curtiss and R.B. Bird, J. Chem. Phys., 74 (1981) 2016.

2 C.F. Curtiss and R.B. Bird, J. Chem. Phys., 74 (1981) 2026. 
3 R.B. Bird, H.H. Saab and C.F. Curtiss, J. Phys. Chem., 86 (1982) 1102.

4 R.B. Bird, H.H. Saab and C.F. Curtiss, J. Chem. Phys., 77 (1982) 4747.

5 H.H. Saab, R.B. Bird and C.F. Curtiss, J. Chem. Phys., 77 (1982) 4758.

6 C.F. Curtiss and R.B. Bird, Physica, 118A (1983) 191.

7 M. Doi and S.F. Edwards, J. Chem. Soc. Faraday Trans., 74 (1978) 1789.

8 M. Doi and S.F. Edwards, J. Chem. Soc. Faraday Trans., 74 (1979) 1802.

9 M. Doi and S.F. Edwards, J. Chem. Soc. Faraday Trans., 74 (1979) 1818.

10 M. Doi and S.F. Edwards, J. Chem. Soc. Faraday Trans., 75 (1979) 38.

11 R.J.J. Jongschaap, 2nd. Conf. of European Rheol. Prague, 1986.

12 R.J.J. Jongschaap and B.J. Geurts, Rolduc Polymer Meeting 2, 1987.

13 B.J. Geurts and R.J.J. Jongschaap, to appear in J. Rheol., May 1988.

14 P.G. deGennes, J. Chem. Phys., 55 (1971) 572.

15 W. Feller, An Introduction to Probability Theory and its Applications, Vols. 1 and 2, Wiley, 1957.

16 A.C. Hearn, REDUCE user's manual. Rand Publ. Cp 78, 1983.

17 B.J. Geurts, preprint Twente University, 1987. 